\title{
Origin of life by careful reading
}

\section{A small meeting last week on the origin of life pinned down some important questions and showed how many more remain unanswered; a more systematic search of the literature for clues would help.}

ON the reductionists' agenda, the origin of terrestrial life necessarily has a central place. If the nature of the world we live in is determined simply by its constituents and the laws of physics, a demonstration that it is possible for self-replicating organisms to arise spontaneously may be the only way of countering the common scepticism that nothing as marvellous as, say, the intricacies of the human eye (let alone brain) could have arisen "by chance" - the derogatory shorthand for "evolution by natural selection". How well are the reductionists doing?

A prior caveat is important: to keep scepticism at bay, the reductionists do not have to recreate life as it exists on the Earth. It would suffice to show that organisms capable of replicating themselves could plausibly have arisen from primaeval inorganic matter. Indeed, it would then be possible to proclaim that our form of life is merely one of several, which is probably the case.

That, of course, is not a novel thought. More than 30 years ago, the late Zdenek Kopal, in his inaugural lecture as the University of Manchester's first professor of astronomy, committed himself to some speculations about the likelihood that radioastronomers would find evidence of person-like life elsewhere and stimulated from Dr Thomas Gold the memorable stagewhisper, "Bloody fool, they might be like rocks!", which was meant as a reference to the place of silicon as the next member after carbon in Group IV of the Periodic Table.

But reconstructing the origin of forms of life not based on carbon must be a much more difficult (or, certainly, a more imaginative) task than that of working out the history of that with which we are increasingly familiar. Indeed, with a little luck, the history may already be written in the genomes of the organisms now extant. For at least three-quarters of the time since molecular biology came of age, people have been remarking on the way in which particular sequences of amino acids and nucleotides are conserved from one species to another, suggesting that even small changes have fallen foul of natural selection and thus that the function of the sequences is primaeval. When it is possible to compare the entire genome sequences of very different organisms and, in particular, to understand the function of the "junk" DNA they carry, who will be surprised if the junk proves to be at least a partial record of past evolution?

Meanwhile, present clues to past events are still painfully sparse. So much was apNATURE · VOL 367 • 3 FEBRUARY 1994 parent from last week's meeting at the Ciba Foundation in London, held on the back of the Royal Society's meeting on planetary science and therefore given to marvelling at the formation of hydrocarbons or their chemical derivatives in various kinds of molecular clouds. Is that where our life began? Sir Fred Hoyle might like to think so, but panspermia remains an implausible hypothesis, and (by Occam's razor) a needless one as well.

Two independent strands of evidence do however pin down the interval during which life began on the surface of the Earth. The dramatic proof provided by the Apollo rocks of major meteoritic impacts on the surface of the Moon between 4.1 billion and 3.9 billion years ago, mirrored by less direct proof that Mercury and Mars suffered similarly, suggests that the Earth must also have been sterile by the time the bombardment ended. But palaeontologists who have learned to recognize agglomerations of single-celled organisms in Archaean rocks are confident that life of some kind was flourishing as quickly as between 200 and 400 million years later. By the uncertainties that attend such ancient dates, that is almost like having an age for the origin of life.

An interesting side-issue arose briefly last week: if the Earth was populated with life so soon after it had been meteoritically sterilized, why did three billion years then go by before fossils of creatures with differently specialized cells began appearing in the fossil record, whereupon there was a huge explosion of diversity? That objection can be met in two ways. First, there was a lot going on. Single-celled organisms can be as complicated as jellyfish, slime moulds and fungi. As someone put it last week, "they had to learn all that chemistry". But who, in any case, says that the acquisition of the biochemical machinery of differentiation and sexual reproduction, the origin of biodiversity, would have been child's play?

So what were the first forms of life really like? As yet, nobody can know. In the half century since Harold Urey and Stanley Miller at the University of Chicago sparked electric discharges through mixtures of water, hydrogen, methane and ammonia (to simulate lightning in the primaeval atmosphere) and made small quantities of amino acids and purines, it has been reasonable to suppose that at least the essential building blocks of life would have been accessible. But the first self-replicating entities must have been molecules, not organisms in the ordinary sense. To escape the dead hand of equilib- rium thermodynamics, they must have been autocatalytic molecules.

That is why the discovery (in the 1970s) of catalysis by molecules of RNA has been such a stimulus for those working in the field. Indeed, the first self-catalysed replicating system, due to Gunter von Kiedrowski in 1986, consisted of the linkage of two trinucleotide molecules catalysed by the hexanucleotide that is the product of the linkage. This is what Leslie Orgel has called "informational" autocatalysis (see Nature 358, 203; 1992), but he also explains why it is unlikely to be extendable to longer RNA molecules; the template and its product would not dissociate.

With all that said, the literature these days seems full of pointers to what life may have been like soon after it began. Thus it is an article of faith that the activity of modern genes is regulated by the interaction between proteins and the relevant DNA, which prompts the question of the primaeval role of these interactions. Then there are ribosomes, the repository of more than 90 per cent of the RNA in a typical cell, which now regulate the translation of nucleotides, three units at a time, into protein residues; what were their primaeval functions?

Several articles in this issue of Nature, as in almost any other, may be clues to what primaeval life was like. Quite apart from the self-assembling double helix organized around sodium ions (page 441) and the interactions between the genes called evenskipped and wingless and between wingless and engrailed (pages 429 and 460) in several insect orders (all long post-primaeval), the tentative structure of the Drosophila transcription factor (page 484), with its six component protein elements, has an Archaean ring to it, especially when account is taken of similarities with the notoriously well-conserved histone proteins. The touchsensitive sodium channel found in the nematode Caenorhabditis elegans, also postprimaeval, like the analogous epithelial sodium channel in the rat kidney, while nicely suggesting how natural selection has led to the formation of a more efficient channel by triplication of a pre-existing gene (pages 463, 467 and 470), is similarly a reminder of the need that the invention of membrane channels should have followed on the heels of that of membranes themselves. Perhaps the next big effort, in the search for the origin of life, should be a careful reading of the journals in the hope of defining the latest dates at which such features could have arisen.

John Maddox 